\title{
Le policier et le culturel. Police et minorités à
} Berlin

Cultural Policing: Police and Minorities in Berlin

Jérémie Gauthier

\section{OpenEdition}

1 Journals

Édition électronique

URL : http://journals.openedition.org/conflits/19577

DOI : 10.4000/conflits. 19577

ISSN : $1777-5345$

Éditeur :

CCLS - Centre d'études sur les conflits lilberté et sécurité, L'Harmattan

Édition imprimée

Date de publication : 30 octobre 2017

Pagination : 123-141

ISBN : 978-2-343-13540-3

ISSN : 1157-996X

Référence électronique

Jérémie Gauthier, «Le policier et le culturel. Police et minorités à Berlin », Cultures \& Conflits [En ligne], 107 | automne 2017, mis en ligne le 30 octobre 2019, consulté le 30 mars 2021. URL : http:// journals.openedition.org/conflits/19577; DOI : https://doi.org/10.4000/conflits.19577 


\section{Le policier et le culturel Police et minorités à Berlin 1}

\section{Jérémie GAUTHIER}

Jérémie Gauthier est sociologue à l'IRIS-EHESS et au Centre Marc Bloch de Berlin. Ses recherches sont consacrées à la force publique et aux usages ordinaires de l'État dans une perspective comparative.

«Dans la société actuelle, nous devons apprendre à composer avec une hétérogénéité, des cultures et des religions différentes. Cela exige d'une part plus de tolérance, et d'autre part une reconnaissance de la loi et des valeurs de notre Constitution par toute personne vivant ici [...] afin que l'intégration puisse réussir. Nous le réalisons, lorsqu'il $y$ a lieu, avec les moyens classiques de la police et de la justice. Mais le point de départ pour le dialogue, le travail en réseau et la confiance, c'est la prévention. Un dialogue sur le long terme se nourrit de communication réciproque et de connaissances sur les autres cultures et religions, nécessite la capacité de reconnaître et de prendre en considération les différences pour surmonter la distance de l'altérité [...] Tout ceci est particulièrement nécessaire dans une période où dans notre pays, plutôt qu'un débat pragmatique sur les possibilités d'intégration, a été menée une discussion islamophobe, belliqueuse entre les cultures et nourrie d'exagérations, de simplifications et de bêtises sur les migrants et les Musulmans".

Dieter Glietsch, Préfet de Police de Berlin, Conférence fédérale de police sur le thème " Migration et Intégration ", 26 octobre 2010

1. L'auteur souhaite remercier les relecteurs ou relectrices anonymes pour leurs précieux commentaires sur ce texte. 
$\mathrm{D}$ ans ce discours prononcé devant plusieurs centaines de policiers allemands et étrangers, journalistes et chercheurs, le préfet de police de Berlin 2 (membre du SPD) met en avant deux aspects spécifiques de l'action policière à Berlin : d'une part, l'approche préventive, mise en œuvre depuis les années 1990 et, d'autre part, à partir des années 2000, son volet «interculturel » à destination des étrangers et des Allemands issus de l'immigration. La rencontre de ces deux logiques a donné lieu à une doctrine appelée « prévention interculturelle » dont l'institutionnalisation est l'objet de cet article. Cette stratégie est présentée par la police, d'un côté, comme un moyen de réaliser l'intégration des étrangers et des Allemands issus de l'immigration dans la société allemande, de lutter contre le racisme et les discriminations et de « diversifier » les forces de police ${ }^{3}$; d'un autre côté, comme un moyen de contrôle des communautés de migrants et de collecte d'informations sur ces dernières. Surprenant d'un point de vue français, le ton très politique du préfet de Berlin traduit également l'importante autonomie dont disposent les polices régionales en Allemagne ${ }^{4}$. Il répond en effet à la chancelière Angela Merkel qui avait déclaré l' «échec du Multikulti 5 » ainsi qu'à l'essayiste Tilo Sarrazin, encarté au SPD et ancien membre du directoire de la Bundesbank, qui avait publié quelques mois avant la conférence un essai xénophobe âprement débattu en Allemagne dénonçant les politiques d'intégration et les dangers que représenteraient l'islam et les Musulmans pour la société allemande ${ }^{6}$.

La prévention policière et son pendant culturaliste sont en Allemagne le fruit d'évolutions fédérales et régionales. Depuis la fin des années 1970, les politiques d'intégration mises en œuvre par le Sénat de Berlin s'appuient sur la diversité culturelle, linguistique et religieuse. Ce cadrage culturaliste de l'action publique, qui a émergé en réaction à des formes de traitement des étrangers jugées trop paternalistes, a concerné en premier lieu les administrations scolaires, les services sociaux et les offices des étrangers avant de toucher la police. Il s'agissait alors de faciliter l'accueil des étrangers dans les administrations et de diffuser des " compétences interculturelles » au sein des personnels ${ }^{7}$. À partir de la loi sur l'immigration de 2000 8, ces politiques d'intégra-

2. Dieter Glietsch a été Préfet de police de Berlin entre 2002 et 2011.

3. Gauthier J., «Des corps étrange $(\mathrm{r})$ s dans la police ? Les policiers minoritaires à Paris et à Berlin », Sociologie du Travail, vol. 53, n4, pp. 460-477.

4. La Loi fondamentale de 1949 attribue en effet aux Länder la définition et la mise en ouvre des politiques de sécurité publique.

5. «Angela Merkel declares death of German multiculturalism », The Guardian, 17 oct. 2010.

6. Sarrazin T., Deutschland schafft sich ab: Wie wir unser Land aufs Spiel setzen, Munchen, Deutsche Verlags-Anstalt, 2010.

7. Schröer H., Interkulturelle Öffnung und Diversity Management. Konzepte und Handlungsstrategien zur Arbeitsmarktintegration von Migrantinnen und Migranten, München, Interkulturelle Qualitätsentwicklung, 2007, p. 11.

8. «La réforme, telle qu'elle est entrée en vigueur au 1er janvier 2000, prévoit ainsi que la nationalité allemande est automatiquement accordée, dès la naissance, aux enfants de la deuxième génération de l'immigration, dont les parents sont eux-mêmes nés à l'étranger, à condition que ces derniers aient depuis au moins huit ans leur résidence habituelle en Allemagne et dis- 
tion connaissent une montée en puissance à l'échelle fédérale puisque les immigrés et leurs descendants ne sont plus considérés comme des «étrangers » à la communauté ethnique allemande, mais comme porteurs d'une "diversité culturelle » perçue comme un « potentiel ${ }^{9}$ ». Ce référentiel d'action publique, qui connaît un important succès à Berlin dont les administrations deviennent le modèle du Multikulti en Europe 10, est adopté par la police berlinoise au début des années $2000{ }^{11}$ sous le nom d' " ouverture interculturelle » (interkulturelle Öffnung der Polizei). Par ailleurs, les émeutes françaises de 2005, interprétées par les cadres policiers berlinois comme le résultat d'une intégration défaillante des descendants d'immigrés, ont conforté la légitimité de cette doctrine.

Parallèlement à la réforme du droit de la citoyenneté et des politiques d'intégration, la définition de l'islam comme problème public connaît un changement d'échelle. Après les attentats du 11 septembre 2001, l'État fédéral cherche en effet à organiser politiquement le culte musulman et à intervenir dans les milieux fondamentalistes, par le biais des services de renseignement notamment (Verfassungsschutz). Toutefois, conformément au droit allemand, la gestion des rapports entre la puissance publique et les communautés religieuses reste majoritairement du ressort des communes et des Länder ${ }^{12}$. Dans ce contexte, les groupes perçus comme musulmans constituent donc une cible centrale dans la stratégie « interculturelle » de la police de Berlin qui, comme on le verra plus bas, va tenter d'investir le registre de la régulation du culte et celui de la gestion sécuritaire.

Ce référentiel culturaliste a été intégré aux stratégies policières dites de prévention « nouveau style 13 ». Définie au niveau fédéral comme un « travail de prévention proche du citoyen à l'échelle communale ${ }^{14}$ ", l'approche préventive a modifié en profondeur le cadrage et la mise en oeuvre des politiques de sécurité. La prévention repose sur deux piliers principaux. Tout d'abord, les décisions sont prises au niveau régional et communal, tandis que le quar-

posent d'un titre de séjour permanent ». Schmid B., «L'Allemagne instille du droit du sol », Plein Droit, 2001, p. 49.

9. Putz R. et M. Rodatz, " Kommunale Integrations- und Vielfaltskonzepte im Neoliberalismus. Zur strategischen Steuerung von Integration in deutschen Großstädten », Geographische Zeitschrift, vol. 101, n³ et 4, 2013, pp. 166-183.

10. Bocquet D. et P. Laborier, Sociologie de Berlin, La Découverte, Paris, 2016, p. 55.

11. Même si certaines initiatives, comme le recrutement de " policiers issus de l'immigration " à partir de 1994, ou encore le développement de l'action préventive de certaines unités spécialisées, ont trouvé un relais dans la diffusion de la doctrine de l'ouverture interculturelle.

12. de Galembert C., "La gestion publique de l'islam en France et en Allemagne : les modèles nationaux à l'épreuve ", in Kastoryano R. (dir.), Les codes de la différence, Paris, Presses de Sciences Po, 2005, pp. 175-202.

13. Aden H., «L'État protecteur, mobilisation de nouveaux acteurs et repli sécuritaire. Les politiques de sécurité et de prévention en Allemagne dans les années 1990 ", Déviance et Société, vol. $25, \mathrm{n}^{\circ} 4,2001$, pp. 459-477.

14. Pütter N., "Polizei und kommunale Prävention - Zwischen Legitimationspflege und vernetzter Repression », CILIP/Bürgerrechte und Polizei, vol. 86, n¹, 2007, pp. 41. 
tier est quant à lui le niveau d'intervention principal ; l'échelon fédéral joue donc un rôle secondaire. Ensuite, la prévention repose sur l'idée de coproduction de la sécurité, c'est-à-dire d'enrôlement d'acteurs publics, semi-publics et privés dans le travail policier. Pour ce faire, des « conseils de prévention » destinés à faire la médiation entre la police, la commune et les citoyens ont été mis en place. La prévention constitue enfin un discours d'institution visant à légitimer l'action policière par la mise en avant d'un souci de défense de la démocratie. Le style de police berlinois promeut donc une définition du rôle du policier orienté vers la régulation sociale plutôt que vers la lutte réactive contre le crime.

Cet article propose d'analyser l'articulation entre les politiques de prévention policière et l'adoption du référentiel culturaliste d'action publique. On y défendra l'hypothèse que l'institutionnalisation du traitement culturaliste des populations minoritaires a eu pour effet un élargissement du mandat policier. En effet, ces nouvelles stratégies et clientèles ont permis à la police d'endosser une fonction de régulation sociale qui va bien au-delà de la simple répression des crimes et des délits. À l'inverse de ce qu'on observe en France, le rapprochement, voire la collusion, entre le travail policier et le travail social ${ }^{15}$ permet à la police d'élargir ses domaines d'intervention notamment par le biais de l'enrôlement de nouveaux acteurs dans le champ de la sécurité publique (mairies, écoles, associations, travail social et représentants communautaires). La police berlinoise a donc su transformer le mouvement réformateur en fenêtre d'opportunité pour élargir son champ d'action à l'échelle locale. Dans le même temps, le référentiel culturaliste produit des effets ambivalents : d'un côté, il atténue l'effet des stéréotypages policiers lors des interventions; d'un autre côté, il produit des formes de réification des groupes étiquetés comme « minorités culturelles ».

L'article se fonde sur des données recueillies dans le cadre d'une enquête ethnographique menée entre 2007 et 2010 dans deux commissariats implantés dans des quartiers berlinois dits « sensibles », ainsi qu'auprès de l'unité «Intégration et migration », qui revendique une spécialisation dans la "prévention interculturelle ». Environ quarante entretiens semi-directifs ont été réalisés avec des policiers et policières de tous grades et plusieurs centaines d'heures d'observation du travail de patrouille ont été collectées. Une autre enquête menée entre 2004 et 2010 dans un commissariat de proche banlieue parisienne (département du Val-de-Marne) sur laquelle se trouvent des cités considérées comme « difficiles » par les policiers permettra d'avancer certains éléments comparatifs franco-allemands. Le choix des terrains en France et en Allemagne a été effectué en raison de leur catégorisation, par les autorités, comme «sensibles»: dans ces secteurs, sécurité et immigration sont constituées en problème public. 


\section{La culturalisation de la prévention}

«Quand on parle aujourd'hui de prévention, on pense très vite aux immigrés, à cette masse critique de jeunes gens et à leurs difficultés d'insertion ».

Gerhart, 54 ans, Chef de l'Unité Intégration et Migration, 2 avril 2008

La prévention policière cible en premier lieu la petite et moyenne délinquance dans les quartiers de Berlin considérés comme difficiles. C’est le cas du quartier d'Antonkiez où l'enquête ethnographique a été conduite et que la presse allemande qualifiait en 2007 de « ghetto », de « quartier chaud » ou encore de « quartier en perdition ». Il s'agit avant tout d'un quartier pauvre, ce dont témoignent notamment les forts taux de chômage et de transferts sociaux 16 ainsi que les habitats dégradés. Comparativement aux autres quartiers de Berlin, les statistiques policières pour l'année 2009 mettaient en lumière un taux élevé de vols, de cambriolages, d'atteintes aux personnes et d'infractions à la législation sur les stupéfiants ${ }^{17}$. Beaucoup de policiers considèrent les habitants du quartier comme appartenant à une classe inférieure (Unterschicht) et mettent en avant la forte proportion d' "étrangers » (Ausländer) et de personnes « issues de l'immigration 》 (mit Migrationshintergund) parmi les habitants. À cette extranéité sont associées par les policiers des formes de déviances spécifiques : les dealers noirs de Grasspark, la criminalité organisée libanaise ou russe, des mosquées de cours d'immeubles organisées en "sociétés parallèles » ou encore les arabes qui seraient plus réticents à se soumettre à l'autorité policière.

Ces représentations policières négatives recueillies lors des entretiens sont à bien des égards comparables à celles recueillies auprès de policiers français lorsqu'on les interroge sur les «banlieues sensibles » et leurs habitants. En pratique cependant, l'action policière observée dans le quartier d'Antonkiez est très différente : le recours aux contrôles d'identité discrétionnaires ainsi que les conflits opposant policiers et habitants y sont en effet rares. Cette relation pacifiée à la police est en grande partie imputable aux stratégies préventives reposant sur le service aux personnes, la résolution de conflits, la médiation et la désescalade, quand les policiers français privilégient au contraire les tâches associées à l'anticriminalité ${ }^{18}$. L'identité professionnelle du policier

16. En 2007, le taux de chômage était de 17,5\% à Antonkiez contre une moyenne de $11,6 \%$ à Berlin. Le pourcentage de personnes bénéficiaires d'un revenu minimum était de 39,1\% à Antonkiez contre $21,8 \%$ à Berlin. Source : [http://www.berlin.de/imperia/md/ content/baneukoelln/allgemeingoedecke/trendanalyse_zusammenfassung.pdf].

17. Kommentierung des Berichts zur Kriminalitätsbelastung in öffentlichen Räumen Berlin 2009, [http://www.berlin.de/imperia/md/content/polizei/kriminalitaet/pks/kommentierung_des_ berichts_zur_kriminalit_tsbelastung_in__ffentlichen_r_umen_2009.pdf].

18. Gauthier J., "Origines contrôlées. Police et minorités en France et en Allemagne », Sociétés contemporaines, vol. 1, n97, 2015, pp. 101-127 et de Maillard J., Hunold D., Roché S., 
berlinois se rapproche alors davantage du travailleur social porté sur la régulation des rapports sociaux dans le quartier que du « chasseur » en perpétuelle quête d'infractions en flagrant-délit.

\section{La prévention: le contrôle sans la force}

« Il y a quelques décennies, les polices en Europe de l’ouest se définissaient de la même manière : un rude métier d'hommes axé sur la répression. Mais ça a changé. La prévention présente beaucoup d'avantages, elle insiste sur la construction de réseaux et sur le vivreensemble (das Wir-Gefübl). Aujourd'hui, la police définit son rôle de concert avec d'autres acteurs. Cependant, la prévention est plus difficile et compliquée que la répression [...] Ça marche s'il y a des idées et le soutien de la hiérarchie ».

Gerhart, 54 ans, Chef de l'Unité Intégration et Migration, Berlin, 2 avril 2008

« La proximité avec le citoyen et la prévention constituent une différence avec Paris. Nous entrons en contact avec nos citoyens, nous essayons de construire des réseaux, nous nous impliquons dans les quartiers. C'est un véritable travail de proximité. Ce que j'ai constaté à Paris, c'est qu'ils n'en sont pas encore là, il y a encore beaucoup de distance avec les citoyens. La police fonctionne encore de manière très militaire. Nous, c'est très différent. Nous sommes la police des citoyens et non une petite armée [...] C'est comme ça que je comprends le terme de "service public" ». Dieter, 55 ans, Commissaire, Antonkiez, 27 septembre 2007

Concrètement, la prévention prend corps dans le travail des policiers de brigades ainsi que dans des unités spécialement dédiées. Au sein des brigades généralistes, les policiers sont tenus de participer aux tâches préventives - les patrouilles pédestres «de proximité », les interventions dans les écoles ou encore la participation aux « équipes anti-conflit 19 » dans les manifestations. La prévention définit également les répertoires d'action policiers déployés lors des interventions : les stratégies de médiation et de désescalade vont être préférées aux contrôles, aux interpellations et aux procédures de garde à vue. À la

Oberwittler D., Zagrodzki M., « Les logiques professionnelles et politiques du contrôle. Des styles de police différents en France et en Allemagne ", Revue française de science politique, vol. 66, n 2, 2016, pp. 271-293.

19. Les Anti-Konflikt Team (AKT) sont des groupes de policiers intervenants lors des événements publics comme les manifestations, les fêtes sur la voie publique (Straßenfest) ou les matchs de football. Leur mission consiste à mettre en place un dialogue avec les organisateurs, les participants, le public, les habitants des quartiers concernés et les médias, afin de prévenir les conflits et de mettre en place des stratégies de désescalade. Les AKT interviennent particulièrement lors d'événements au cours desquels des émeutes sont susceptibles de survenir (comme par exemple les manifestations du 1er mai à Kreuzberg). 
différence du cas français, l'emphase sur la prévention contribue ici à réduire les pratiques de contrôles policiers proactifs 20 . Alors que les policiers français définissent souvent leur identité policière en opposition avec la figure de l'« assistante sociale ", à Berlin au contraire, la dimension sociale du travail policier est légitimée par la politique de prévention et beaucoup de policiers assument, voire revendiquent le rapprochement avec le travail social.

De l'anticriminalité au travail social, la trajectoire professionnelle d'Herbert (voir l'encadré) traduit l'étendue du répertoire d'action policier pouvant être investi au cours d'une carrière. Ainsi, la valorisation par le politique et la hiérarchie de certains types de tâches pourtant a priori éloignées du « vrai travail policier » peut mener à une redéfinition de l'identité professionnelle, des objectifs et de l'efficacité policière. Le contraste est ici saisissant avec la France, où la séparation entre travail policier et travail social avait notamment été consacrée à Toulouse en 2003 par Nicolas Sarkozy, alors ministre de l'Intérieur, pour qui le « respect de l'ordre public » n'était plus censé passer par l'« organisation de matches de rugby » ou «des activités d'assistance sociale ». L'exemple berlinois montre également que la condition policière n'est pas une culture homogène et inclut des représentations différentes, voire antagonistes, du métier, et que c'est au politique qu'il appartient de valoriser symboliquement et matériellement telle identité professionnelle et pratique du métier plutôt qu'une autre.

\section{Herbert, le « policier social »}

Herbert est né à Berlin-Ouest dans les années 1960 d'un père ouvrier et d'une mère femme au foyer. II entre dans la police à 17 ans car il souhaite " aider les autres », estime que " cette démocratie vaut le coup d'être défendue " ainsi que pour le salaire intéressant. À l'issue de sa formation, Herbert est affecté dans le quartier de Spreeheim en 1980 où il intervient lors des émeutes du 1 er mai. Il découvre également une population immigrée qu'il n'avait pas l'habitude de fréquenter. D'après lui, à cette époque, les policiers intervenaient sans se soucier des différences culturelles et des manières de vivre.

C'est, selon Herbert, à la fin des années 1980 que la police a eu affaire " aux premiers jeunes issus de l'immigration qui sont sortis des rails, qui ne se sentaient plus soutenus par la société et qui faisaient des conneries». Selon Herbert, àcette époque, beaucoup d'enfants des pays arabes sont arrivés, notamment des Libanais, dont les familles se regroupaient dans des immeubles communs. Autour des familles se serait développée une criminalité faite de rapine, de vols et de violences.

En 1989, Herbert intègre la brigade anti-criminalité d'Antonkiez. Au cours des années 1990, il considère que la situation du quartier s'est fortement détériorée en raison de la constitution de bandes et de l'évolution de nombreux jeunes vers des formes de criminalité organisée. En réaction,

20. Gauthier J., 2015, op. cit. 
Herbert et ses collègues sont "massivement intervenus » contre le développement de la "violence des groupes de jeunes », notamment en patrouillant de manière régulière sur les lieux de rendez-vous des bandes. Mais, selon Herbert, ce type d'intervention de la police n'est efficace que si d'autres institutions prennent le relais. C'est selon lui comme ça que s'est constitué un réseau d'intervention sur un micro-quartier sensible d'Antonkiez et qu'ils ont gagné le "Prix de la prévention " pour leur travail en réseau. Herbert, qui effectue ses tâches de prévention en civil, se définit comme " policier social » (Sozialpolizist), le social n'étant pas la « tâche originelle de la police, mais dans beaucoup de secteurs, nous sommes le dernier recours ". Selon Herbert, il reste encore « quelques idiots " qui critiquent la prévention mais beaucoup ont compris que ça apporte beaucoup personnellement, permet de développer de nouveaux concepts et apporte une satisfaction au travail supérieure à celle du travail normal en brigade.

\section{La culture comme catégorie d'action policière}

Au sein du dispositif de prévention, le volet « interculturel » est compris comme une valeur ajoutée au travail policier pour traiter ce qui est perçu comme relevant de problèmes spécifiques aux populations migrantes. Ce cadrage culturaliste est présenté par les policiers enrôlés dans la politique d'interculturalité comme un savoir supplémentaire, censé améliorer le discernement des agents, accroître l'efficacité des interventions et lutter contre les préjugés raciaux. Les «problèmes » entre policiers et personnes issues de l'immigration sont interprétés à travers des référents culturels, aussi bien par les policiers de la «base » engagés dans les tâches préventives que dans la politique institutionnelle d'" ouverture interculturelle » de la police berlinoise. Du point de vue des policiers, la résolution des « conflits culturels » passe par la compréhension de ces «cultures immigrées », comme c'est le cas lors de l'intervention ci-dessous.

Vers 22h45, j'accompagne Martin et Roni suite à un appel pour agression. Lorsque nous arrivons à l'adresse indiquée, une jeune fille, Zahra, nous ouvre. Elle est d'origine libanaise, a environ 18 ans et parle parfaitement allemand, à l'instar de ses frères et sœurs. Dans le salon, où se trouvent de nombreux bibelots portant des inscriptions en arabe, se trouvent deux petits garçons, un adolescent, une adolescente, leur mère (qui porte le foulard, parle mal l'allemand et a l'air fatiguée) ainsi qu'une autre femme en train de fumer.

Zahra a appelé la police car son père, séparé de sa mère, s'est rendu à leur domicile pour les menacer. Zahra et sa mère ne l'ont pas laissé entrer. D'après la jeune femme, son père l'avait promise en mariage à un cousin lorsqu'elle avait 16 ans. Elle dit que son père "pense encore comme au Moyen-Âge ». Roni, qui m'avait fait part dans la 
voiture de ses préjugés envers les «Arabes », lui répond que "c’est le cas de beaucoup de pères arabes à Berlin ». Puis il demande au grand frère de témoigner et ils vont s'enfermer dans la cuisine. Les deux policiers souhaitent ensuite parler à la mère. Zahra précise que sa mère « ne comprend rien à l'allemand ». Zahra traduit donc les questions du policier en arabe et les réponses de sa mère en allemand. La mère a l'air perdue, ce qui contraste avec la vivacité de Zahra et des enfants.

Je me rends ensuite en bas de l'immeuble où les policiers de la brigade anti-criminalité (StrDVB), arrivés après Martin et Roni, discutent avec le père de Zahra, un de ses amis et deux hommes que les policiers me présentent comme des « agresseurs ». Le père, qui a un œil tuméfié, ne parle pas très bien allemand. Il est calme et déférent. Son ami est déférent lui aussi et parle mieux l'allemand. Il dit qu'il souhaitait uniquement voir ses enfants et qu'il « se fout » de la mère. Il précise également qu'il lui importe peu qui épouse sa fille : " même un Allemand! ". Comme Zahra et sa mère ne l'ont pas laissé entrer, il est resté sur le trottoir. Il comptait partir mais il s'est alors fait «agresser » par deux cousins de la mère. L'un d'eux l'a frappé à l'œil avec une ceinture. Soudain, deux hommes libanais surgissent et l'un d'entre eux commence à crier en arabe vers le père et à le menacer physiquement. Les policiers contiennent les deux parties. Roni et Martin, qui sont redescendus après avoir enregistré la plainte de la mère, invitent le père à déposer plainte contre ses agresseurs. Au bout d'un moment, Roni clôt la discussion : «je ne veux pas que ça mène à une bataille générale entre vos deux familles! ». Le père approuve mais le policier lui rétorque que « l'expérience montre que c'est souvent le cas ». Dans la voiture, Roni me dit «Oui, c'est souvent comme ça, les pères veulent faire comme avant et les jeunes ne veulent plus ! ». Puis, en soupirant d'un air blasé : «C’est toujours compliqué avec les Arabes!".

Extrait du journal de terrain,

Antonkiez, 5 octobre 2007

Lors du débriefing dans la voiture, Martin et Roni interprètent cette intervention comme un « conflit culturel » lié à la pratique du mariage forcé, aux différences linguistiques et générationnelles entre les parents d'origine turque ou arabe et les enfants nés et socialisés en Allemagne. Le fait que « ce soit toujours compliqué avec les Arabes » traduit une mise à l'épreuve de l'action policière par des types d'interventions interprétées sur le registre de la différence culturelle. Dans cet extrait, la frontière entre le « eux » et le « nous » divise avant tout les générations : aux yeux des policiers, si les parents sont décrits comme des « étrangers » culturellement différents, les enfants, et notamment la jeune femme, sont perçus comme des Allemands « issus de l'immigration ». 
Ce cadrage culturaliste de l'intervention par les policiers, nourri par les «compétences interculturelles » acquises lors des séances de formation, a un effet de régulation de l'action policière en ce qu'il rompt le lien entre représentations et pratiques policières. L'hostilité de Roni envers les «Arabes », maintes fois affirmée dans le huis clos policier, ne se traduit en effet pas dans ses interactions avec le public où un savoir-faire culturaliste se substitue aux stéréotypes individuels.

\title{
L'islam comme laboratoire de l'"interculturalité » policière
}

Le cadrage culturaliste de l'action policière ne concerne pas uniquement les unités de police-secours. Il s'inscrit plus largement dans une stratégie de contrôle de l'islam et des groupes étiquetés comme «musulmans ». Redéfinie dans des termes religieux, la prévention interculturelle vise alors à enrôler des acteurs communautaires dans la prévention de la délinquance, à produire un savoir policier sur l'islam et à surveiller les communautés musulmanes.

\begin{abstract}
« À Antonkiez, il y avait une mosquée dans l'arrière-cour d'un immeuble, comme c'est souvent le cas. Et des résidents se sont inquiétés car ils ne savaient pas ce que c'était, qui vient là, etc. Il y avait un manque d'information et donc des peurs. L'Unité Intégration et Migration avait commencé à enquêter. Dans le même temps, un groupe de jeunes occupait l'espace public avec des chiens et importunait les gens. Mais la situation sécuritaire objective en termes d'infractions n'était pas très préoccupante, seulement quelques délits classiques : vols, dégradations, petites agressions. Nous sommes entrés en contact avec la mosquée par l'intermédiaire de l'organisation-mère de la communauté turque en leur demandant s'ils étaient prêts à coopérer avec nous. Ils ont rapidement accepté et nous avons demandé à la mosquée de nous mettre en relation avec ces jeunes qui causaient des problèmes devant leur porte : "peut-être pouvez-vous utiliser votre potentiel d'influence sur les membres de la communauté, sur les parents ou bien directement sur les jeunes, et peut-être atteindre quelques objectifs avec la police ?" Mais il ne s'agissait pas uniquement de jeunes appartenant à leur communauté, il y avait aussi des Allemands parmi eux, ce que nous avons pris en compte. La brigade chargée du secteur a un peu enquêté. Et les gens de la mosquée ont proposé un local pour que les jeunes reçoivent de l'aide aux devoirs, fassent un peu de sport et d'informatique. Et peu à peu, nous avons obtenu le calme dans ce secteur ».
\end{abstract}

Dieter Schwarz, responsable du projet TIK, 28 avril 2010

L'« ouverture interculturelle » commençait alors à se préciser dans des termes policiers : il s'agissait d'obtenir d'acteurs identifiés comme pertinents 
(ici, les mosquées) qu'ils coopèrent avec la police pour atteindre des jeunes perçus comme membres de la communauté musulmane de Berlin. Pour les responsables policiers, il fallait donc impliquer des acteurs communautaires dans le travail policier en mettant en avant les notions de «dialogue », de « confiance » et de " projets communs ». La prévention interculturelle se traduit donc dans les faits par une forme de "community policing », bien que ce terme ne soit jamais employé par les acteurs.

La prévention interculturelle prend également pour cible l'islam radical. Le chef de l'Unité Intégration et Migration, dont le travail sera évoqué plus bas, définit son unité comme un maillon de la lutte contre les fondamentalistes qui « agissent à l'encontre des valeurs fondamentales et qui essaient de détruire l'ordre démocratique ». Il personnalise par ailleurs l'action de son unité dans un contexte élargi : "je ne souhaite pas que ce pays ou l'Europe soit islamisé(e) ! ». Il constate également la progression des «tendances islamistes », notamment par l'action de fondamentalistes qui essaieraient de former des « petits » à l'islam radical. Il insiste enfin sur le « ralliement récent des enseignants au travail policier » suite à un « appel au secours » lancé par différentes écoles du quartier en direction du politique et des administrations. D'où l'attention particulière que porte son unité sur les écoles du quartier d'Antonkiez en intervenant lors de conflits qualifiés d'interculturels afin de "fournir des arguments sur les croyances religieuses » là où les enseignants sont perçus comme défaillants. Le chef de l'AGIM milite ainsi pour une hausse du niveau de l'enseignement de l'islam dans les écoles, ainsi que pour une levée de l'interdiction du voile dans le corps des enseignants - les jeunes femmes musulmanes étant selon lui les «meilleures interlocutrices » en cas de conflit. Cette revendication d'un discours expert sur l'islam constitue une ressource centrale pour les policiers impliqués dans la prévention interculturelle qui leur permet de se distinguer au sein de la profession tout en revendiquant une spécialisation dans le contrôle des minorités culturelles.

\section{Les «spécialistes de l'intégration "}

Les policiers de la brigade spécialisée unité Intégration et migration (Arbeitsgebiet Integration und Migration, UIM), nommée jusqu'en 2009 Unité Étrangers (Arbeitsgebiet Ausländer), se présentent comme les «spécialistes de l'intégration ». Compétente sur les quartiers de Spreeheim, Antonkiez (ex-Berlin-ouest) et un quartier de la partie est de la ville, cette unité constitue la principale entité sur laquelle repose l'institutionnalisation de la prévention interculturelle dans la police berlinoise et son évolution reflète la transformation récente du rapport de la police aux populations issues de l'immigration. Atypique au sein de l'organisation policière, l'UIM revendique ses « compétences interculturelles » pour se démarquer des tâches policières traditionnelles. L'analyse de l'UIM comme instrument ${ }^{21}$ de l'institutionnali- 
sation de la prévention interculturelle dans la police berlinoise permet donc d'étudier la marge de manœuvre dont disposent des acteurs policiers pour redéfinir leur rôle au sein de la profession et légitimer une action atypique et contestée en interne.

\section{De la répression des étrangers au contrôle social des minorités}

«La police ne se définit pas par la force mais par l'importance de ses réseaux. On n’a pas besoin du nombre quand on a la connaissance ». Gerhart, 54 ans, Chef de l'Unité Intégration et Migration, 2 avril 2008

Jusqu'aux années 1970, la population étrangère de Berlin-Ouest était essentiellement constituée de «travailleurs invités » turcs dont le recrutement sous contrôle des autorités allemandes avait pour objectif de satisfaire les besoins du marché du travail, d'éviter le regroupement communautaire et, pensait-on alors, d'anticiper le retour au pays une fois le besoin de maind'œuvre tari. Cependant, le début des années 1970 voit l'arrêt du principe de rotation des travailleurs migrants ainsi qu'une augmentation du nombre de migrants originaires de pays hors-CEE 22. Parallèlement, la crise économique de 1973 conduit à une politique de fermeture des frontières et de restriction des renouvellements de permis de séjour. Dans ce contexte, les autorités de la ville de Berlin tentent de recenser les immigrés sans titre de séjour. Or, à l'époque, la police berlinoise est dépourvue d'agents compétents dans le domaine du droit des étrangers. Le gouvernement socialiste de Berlin et la Préfecture de police proposent alors de créer une unité chargée de traiter cette problématique d'un " point de vue policier ", afin de réguler, voire de mettre fin aux entrées et aux séjours irréguliers sur le territoire ouest-berlinois. Des policiers volontaires sont affectés à la première mouture de cette police des étrangers qui porte tout d'abord le nom de Arbeitsgebiet gezielte Ausländerüberwachung (AGA) c'est-à-dire "Unité spécialisée dans la surveillance ciblée des étrangers ».

Dans un premier temps, beaucoup de migrants sont interpellés à des fins d'identification : la police souhaite déterminer leur origine, leur statut, connâ̂tre les raisons de leur venue à Berlin et décider d'une éventuelle expulsion vers le pays d'origine. La population-cible et les missions de l'AGA sont alors clairement définies : la répression des «illégaux »(Illegalen). Dans le même

21. Un instrument d'action publique constitue un dispositif à la fois technique et social qui organise des rapports sociaux spécifiques entre la puissance publique et ses destinataires en fonction des représentations et des significations dont il est porteur (Lascoumes P., Le Galès P., "L'action publique saisie par ses instruments ", in Lascoumes P., Le Galès P. (dir.), Gouverner par les instruments, Paris, Presses de Sciences Po, 2005, pp. 11-44).

22. Münz R., Ulrich R., "Les migrations en Allemagne : 1945-1996 », Revue européenne de migrations internationales, vol. $14, \mathrm{n}^{\circ} 2,1998$, p. 186. 
temps, l'AGA oriente ses activités vers la répression d'infractions associées aux communautés étrangères : détection des mariages blancs à partir des années 1980, répression du trafic de cigarettes dans les milieux vietnamiens, travail au noir, jeux illégaux et prostitution asiatique et est-européenne. L'appui de l'AGA est également sollicité par des unités de sécurité publique ou de police judiciaire qui souhaitent utiliser ses compétences en matière juridique lors d'interventions policières dans les milieux immigrés de Berlin. Ainsi, la volonté de policer des étrangers dont on ne comprend « ni la langue, ni la culture » n'est perçue comme un enjeu professionnel par les policiers qu'à partir des années 1970 et 1980.

À partir des années 1990, le développement du volet préventif est concomitant de la décrue de l'immigration irrégulière dans les années 1980, entrầnant de fait une baisse de l'activité répressive. Les missions préventives et les étrangers en situation régulière vont donc constituer le nouveau paradigme de l'AGA, qui continue parallèlement son travail répressif visant l'immigration irrégulière. Cette transformation du rôle de l'AGA se traduit en 2009 par un changement de nom de l'unité, rebaptisée Unité Intégration et Migration (UIM). Une figure professionnelle nouvelle va alors émerger : le spécialiste en « compétences interculturelles », dont l'action s'oriente aussi bien en externe, en direction de la population et des acteurs institutionnels, qu'en interne, à destination des différents services de police.

\section{"Nous sommes les spécialistes des migrations, de l'islam, des cultures et des mentalités !»}

Gerhart est le chef de l'Unité Intégration et Migration depuis sa création en 1973. Il perçoit son unité comme le fer de lance de l'interculturalité et ses subordonnés comme des "spécialistes de l'intégration » qui « doivent s'ouvrir sur l'interculturel, c'est-à-dire la religion, la culture et la mentalité des immigrés ». Pour ce faire, Gerhart recrute des policiers qui doivent "savoir parler, être ouverts aux gens, s'intéresser à la politique et à la résolution de conflits ». Selon lui, la compétence principale de son unité tient à la constitution d'un réseau de "partenaires » : écoles, mairies, travailleurs sociaux ou encore associations "culturelles ». Concernant les écoles, il est particulièrement fier que son unité soit à même de leur " enseigner » la complexité de l'islam et de répondre par la médiation et le dialogue à I' " appel au secours » que les enseignants ont lancé. À ce titre, son unité s'intéresse particulièrement à l'islam. Il s'agit d'une part, en interne, d'en comprendre l'histoire, les valeurs et I'organisation (les différents courants par exemple) et de transmettre ces connaissances à leurs collègues d'autres services par le biais de séances de "formation interculturelle »; d'autre part, I'UIM prétend lutter en externe contre les fondamentalistes religieux qui « essaient de détruire les valeurs de l'ordre démocratique ». 
Dans ce contexte où l'institution policière perçoit l'immigration comme un défi à relever, des modules de formation aux « compétences culturelles » ont été mis en place pour modifier les rapports des policiers aux migrants, notamment pour répondre aux formes de racisme qui circulent dans le monde policier. Ainsi, les policiers de l'UIM se doivent de posséder un savoir sur l'histoire migratoire de la ville, sur les différents courants de l'islam, sur l'histoire ou encore les traditions des pays d'origine des migrants. Cette approche culturaliste de la différence a pour objectif de fournir aux policiers des cadres de compréhension des « réactions» des migrants lors des interventions. À ce titre, l'UIM fait office de centre d'(auto-)formation pour ses propres agents ainsi que pour les policiers de services extérieurs dans le cadre du «transfert de compétences interculturelles » (TIK).

\section{Observation d'une séance de "formation interculturelle "}

Dans le cadre d'une séance de deux heures de formation dispensée auprès de vingt-cinq policiers, la policière de I'UIM explique le changement de nom de son unité par le fait que la police a de «moins en moins affaire à des étrangers mais à des personnes issues de l'immigration dont la majorité est musulmane, et que la religion est donc très importante ». Elle insiste sur le fait que "l'Allemagne s'ouvre, que c'est désormais un pays d'immigration et qu'il faut comprendre et s'ajuster aux autres cultures ». Concernant le travail de police, il est important de savoir comment se comporter avec les personnes issues de l'immigration afin d'éviter les conflits et d'améliorer le travail. Elle ajoute que les étrangers sont également les électeurs du futur.

L'essentiel de la séance porte sur l'islam. Les « formateurs en compétences interculturelles » évoquent l'exemple de jeunes filles, bonnes élèves qui, à leur retour de vacances d'été en Turquie, ne veulent plus participer au cours de sport et portent le foulard. L'UIM entre alors en contact avec la mosquée et tente de ramener les filles dans le système scolaire [soupirs indignés de l'assistance]. Une policière demande : «Pourquoi, alors qu'il y a des lois en Allemagne pour aller à l'école, ils commencent à vouloir dépasser les limites? ». Selon la formatrice, quand les "immigrés arrivent ici, ils ne sont pas obligés de parler allemand, les enfants grandissent en turc et les parents ne connaissent pas I'Allemagne (surtout les femmes), ses droits et ses devoirs ». La policière insiste : "Pourquoi on laisse se développer ça à Berlin? Je n'en reviens pas que ce soit le pays d'accueil qui fasse tous les efforts et pas les migrants! ». La formatrice, à bout d'arguments, répond que "l'Allemagne est un pays d'immigration, point final ! ».

Ces séances de "formation interculturelle » peuvent être comprises comme des formes d'étayage institutionnel, c'est-à-dire qu'elles sont destinées à fournir des cadres d'interprétation du fait minoritaire aux policiers de terrain. Cette forme d'étayage institutionnel contraste avec le cas français, où les 
cadres d'interprétation de l'altérité sont laissés par la hiérarchie aux seuls policiers de terrain. La réaction de la policière aux arguments de sa collègue formatrice donne ici à voir les résistances que peut rencontrer le cadrage culturaliste de l'action policière. Néanmoins, à l'instar du cas de Roni évoqué plus haut, ces séances de formation contribuent à différencier les représentations individuelles des policiers et policières de leurs pratiques en intervention.

Un second ensemble de tâches "préventives » s'oriente vers l'extérieur de l'institution policière et concerne le travail de mise en réseau avec, d'une part, des acteurs considérés comme influents au sein des communautés immigrées (les mosquées, les associations culturelles, politiques, religieuses et sportives, les promoteurs de spectacles ou d'événements sportifs) ainsi que, d'autre part, différentes administrations berlinoises (l'Office des étrangers, le Préposé aux étrangers et à l'immigration, les écoles et administrations scolaires ou encore le Sénat de Berlin). Petit à petit, l'UIM s'est construit un véritable réseau de «partenaires » qui lui assure à la fois une réserve d'informateurs et une base de légitimité. En outre, l'UIM met à disposition ses agents lors d'interventions policières ciblées sur les communautés étrangères (contrôle de régularité des maisons closes berlinoises, maintien de l'ordre dans des manifestations sportives ou culturelles, opérations spéciales sur les lieux de trafic de stupéfiants). Enfin, l'UIM est associée par la hiérarchie policière au développement conceptuel et pratique de l'« ouverture interculturelle » des services de police, notamment concernant les mesures de discrimination positive dans le recrutement de policiers et de policières « issus de l'immigration » au sein de la police berlinoise. L'unité se donne aussi comme objectif de transmettre cette " philosophie » aux autres services de police, ce qui ne manque pas de provoquer certaines réticences, voire une franche hostilité. Cette orientation se veut idéaliste dans le discours ("vivre ensemble») et pragmatique dans les faits, puisqu'il s'agit d'établir un contact permanent avec les communautés étrangères afin de maitriser toutes les situations où la police est susceptible d'intervenir.

\section{Cultures et conflits}

Concernant l'islam, l'UIM cherche à se positionner en médiatrice de conflits entre l'administration scolaire et les familles musulmanes. Cette présence dans les murs de l'école constitue une extension du domaine d'intervention de la police là où elle n'était traditionnellement pas la bienvenue. En mars 2008, j'ai eu l'occasion d'observer un conflit qualifié d' « interculturel » à l'occasion duquel les membres de l'UIM ont été sollicités et qui opposait un imam à une direction d'école primaire d'Antonkiez. Le conflit a pour origine un courrier, disponible en ligne, émanant d'une " association culturelle islamique » et concernant une élève de CM2. Le courrier, signé par les parents de l'élève et adressé à la directrice de l'école, a pour objet une « demande de dis- 
pense du cours mixte de natation » concernant la jeune fille ${ }^{23}$, en se réclamant notamment d'une jurisprudence du droit administratif fédéral (BVerwG, 1993, 6C8/91). La directrice de l'école concernée a envoyé une copie du courrier à l'UIM, qui s'est saisie de l'affaire. En effet, les policiers de l'UIM interviennent lorsqu'ils considèrent que l'ordre local est menacé par un « conflit interculturel ». À ce titre, lors de ces «missions de médiation », le chef de l'unité déclare fièrement que son service participe à la «petite politique locale ». Ce travail trouve la plupart du temps à se concrétiser dans les écoles du quartier, qui sont souvent le théâtre de violences ou de conflits entre l'administration, les élèves, les parents d'élèves et les acteurs politiques et religieux locaux.

Nous quittons donc les locaux de la police pour nous rendre à l'école où la directrice a organisé une réunion avec l'imam qui représente l'association islamique. J'accompagne Gerhart, le chef de l'UIM, et Sandra, une de ses subordonnées d'origine turque «pour traduire si besoin ». Les policiers endossent alors l'habit du «médiateur », en civil et sans arme. Gerhart explique que l'UIM s' « occupe » de la mosquée en question depuis plusieurs années et connaît bien l'imam, dont les enfants sont par ailleurs scolarisés dans cette école. Ce jour-là, son but est d'un côté de faire comprendre à la direction de l'école la «complexité » de l'islam et, de l'autre, de faire accepter les règles scolaires à l'imam. Il cite l'exemple d'une conférence à laquelle il a assisté et au cours de laquelle une directrice d'école avait également évoqué les demandes de dispense des cours de natation. D’après Gerhart, "à une époque où il y a autant de musulmans, on devrait savoir exactement quelles sont leurs dispositions envers les jeunes garçons et filles, notamment concernant l'interdiction de natation (Schwimmverbot), et là, on peut réagir professionnellement ». Gerhart considère que l'école aurait dû réagir immédiatement en adressant une plainte aux parents : «Quand un enfant est dans une école publique, il doit en accepter les règles ». D'un autre côté, il estime que le fait pour l'école et la police de connaître les différents aspects de l'islam les rend plus « professionnelles » et permet de «mieux résoudre les problèmes et les conflits ». Il considère enfin que la police est plus apte à intervenir dans ces conflits car « [elle est] déjà plus ouverte interculturellement et [a] davantage réfléchi à ces problèmes que le personnel scolaire ».

Le jour de la réunion, nous nous asseyons autour d'une table avec l'imam (qui représente les parents de la fillette), la directrice de l'école et son adjointe, ainsi que le «chargé des questions de migration » à la mairie d'Antonkiez.

23. Dans ce courrier, les parents se présentent comme des «musulmans pratiquants » considérant la «culture normative » de l'islam comme « obligatoire». Ils rappellent qu'en vertu de ces normes, les garçons et les filles, au plus tard lors de leur entrée dans la maturité sexuelle, sont tenus de dissimuler leurs «parties honteuses » à l'autre sexe. En conséquence de quoi, si une « dame musulmane » veut respecter ces « règles vestimentaires », sa participation au cours de natation est impossible. 


\section{Un « conflit interculturel » (extraits)}

La directrice ouvre la table ronde en précisant que dans le Land de Berlin, son école a une "mission d'enseignement " (Bildungsauftrag) que les enseignants sont en charge d'appliquer : " L'école est une institution d'État, non une institution religieuse ou une mosquée et nous avons organisé une réunion avec les parents pour préciser quelles sont les frontières qui ne doivent pas être franchies».

Le chef de I'UIM intervient et explique pourquoi l'école a demandé une médiation de la police. Il précise qu'il faut prendre en compte l'orientation religieuse des Chiites mais qu'il est difficile pour une école de composer avec toutes les orientations de l'islam.

L'imam, qui parle dans un allemand incertain avec un fort accent, un cheveu sur la langue et recourt parfois à la policière de I'UIM pour la traduction, explique que sa mosquée délivre des certificats aux femmes qui veulent passer le permis par exemple tout en portant le foulard mais, qu'en règle générale, il ne le fait pas pour les enfants scolarisés : "Je sais que nous ne devons pas nous immiscer dans le domaine scolaire. Mais souvent, des parents me demandent: "Mon enfant a des problèmes, elle veut porter le voile mais l'enseignant ne veut pas, que dois-je faire ?" ". II précise que pour les Chiites, les filles doivent porter le voile à partir de 10 ans, cacher leur "peau et leur parties honteuses».

La directrice poursuit : "Si on ne va pas dans votre sens, qu'allez-vous faire? Dire que nous sommes xénophobes?».

L'imam : "Rien! Mais [la fille] devra trouver une autre école !».

La policière de I'UIM : "Donc vous enverrez cette fille dans une autre école?».

Le chargé des questions de migration : "Comme en France ! ».

[Tous se tournent vers moi qui ne trouve rien à dire].

Le chef de I'UIM : "Nous ne sommes pas en France ! On ne trouve pas une solution isolément! Vous devez aider l'école, les parents et la fille à trouver une solution commune ! Ici, nous ne faisons pas de la grande politique, mais la petite politique d'Antonkiez!»

Suite à cette réunion, les policiers de l'UIM vont « suivre l'affaire ». Dans ce cas, elle se soldera par un succès de leur point de vue car la jeune fille ne changera pas d'école. Cette réunion montre les tensions existantes à l'échelle locale entre les représentants de différentes autorités (scolaire, religieuse et municipale) et la façon dont se posent les « conflits culturels » : les normes majoritaires sont mises à l'épreuve par des normes minoritaires. Dans ce cas, la direction de l'école fait appel à des acteurs municipaux et policiers pour trouver une issue au conflit. En s'investissant dans la « résolution de conflits culturels ", les policiers de l'UIM investissent un rôle politique par la mise en avant de leurs "compétences interculturelles » et leur «travail en réseau " auprès des représentants cultuels musulmans. L'élargissement du domaine de 
compétence policier à l'« interculturalité » ouvre ainsi aux policiers une fenêtre d'opportunité pour étendre le périmètre de leur action. Cette évolution du rôle de la police reconfigure le jeu d'acteurs de la politique du quartier entre la justice, l'institution scolaire, l'Office de la jeunesse (Jugendamt), l'Office des étrangers (Ausländerbehörde), les associations de migrants et la mairie au profit de la police. Cet élargissement du rôle de la police provoque néanmoins des conflits d'autorité entre les institutions. Suite à la réunion évoquée plus haut, les policiers de l'UIM, la directrice de l'école et le chargé des questions de migration ont en effet évoqué la question du transfert d'autorité : en recourant à la médiation policière, la directrice doit déléguer une part de son autorité aux policiers devant un acteur externe, l'imam. Elle souhaite que, sur le long terme, les problèmes puissent être traités exclusivement au sein de l'école. Selon le chargé des questions de migration pourtant, l'«autorité est pour le moment en faveur de la police ».

Cette transformation du rôle traditionnel de la police de sécurité publique a également des effets sur les acteurs «partenaires» de l'action policière. Par exemple, la «coopération » des associations de migrants avec les policiers de l'UIM, ainsi qu'avec les policiers de commissariat impliqués dans les tâches préventives, peut ouvrir à une reconnaissance de statut ainsi qu'à l'attribution de subventions municipales. Les représentants de ces associations ont ainsi intérêt à entretenir de bonnes relations avec l'institution policière. Par ailleurs, on peut faire l'hypothèse que cette « économie de l'interculturalité » influe sur la manière par laquelle les différents acteurs vont construire leur « identité culturelle».

Angela Merkel avait donc annoncé la « fin du Multikulti » de manière prématurée. La police de Berlin s'est au contraire approprié le cadrage culturaliste et l'a transformé en ressource pour asseoir son rôle dans le contrôle social et élargir sa marge de manœuvre face aux autres institutions. Du point de vue policier, l'investissement dans les tâches perçues comme relevant du travail social peut donc constituer une fenêtre d'opportunité. Vu de France, où la dimension sociale du travail policier est dévalorisée et considérée comme une faiblesse au profit de l'usage de la coercition, le contraste est saisissant. Si, en France comme en Allemagne, les groupes minoritaires, et parmi ceux-ci les jeunes hommes, constituent une cible privilégiée de l'action policière, les politiques policières menées à Berlin conduisent à la mise à distance des pratiques policières les plus coûteuses en termes de coercition et de discrimination, comme par exemple les contrôles d'identité discrétionnaires. Ainsi, le détour par le cas berlinois montre qu'une police peut procéder au contrôle social sur des territoires et des populations sans pour autant recourir à un usage accru de la force et de la coercition. 
Cet élargissement du soft power policier pose néanmoins un certain nombre de problèmes. Comme le note le sociologue britannique Michael Banton, la « police n'est qu'une agence de contrôle social parmi de nombreuses autres 24 ». À ce titre, des réserves s'imposent lorsque la police élargit son exercice du contrôle social. Tout d'abord, la participation des administrés à la définition de l'action de la police reste très marginale et on manque de données objectives sur les éventuels gains de légitimité engrangés par la police auprès des gouvernés, et parmi eux les groupes ciblés comme " minorités culturelles ». Plus encore, cet élargissement du rôle de la police renforce le pouvoir de définition policier des problèmes sociaux au détriment d'autres formes de problématisation. De plus, les administrés et les groupes cibles de l'action policière tendent à être perçus comme des sources d'information et comme relais de la volonté policière.

Enfin, et c'est une limite de l'enquête ethnographique conduite à l'intérieur de l'institution policière, il faudrait pouvoir ajouter à l'analyse le point de vue des groupes étiquetés comme "minorités culturelles » et les effets de l'approche culturaliste sur ces derniers. Ainsi, si la police berlinoise apparaît moins génératrice de discrimination que dans le cas français ${ }^{25}$, elle n'en contribue pas moins à étiqueter des sous-groupes de la population sur la base de la culture ou de la religion ${ }^{26}$. Afin de prolonger cette analyse, il faudrait renverser la perspective afin de voir en quoi ce type d'actions policières contribue à prescrire une identité minoritaire du côté des acteurs concernés et peut éventuellement mener à des formes de profilage culturel.

24. Banton M., The Policeman in the Community, London, Tavistock, 1964, p. 1.

25. Gauthier J., 2015, op. cit. et de Maillard J. et al., 2016, op. cit.

26. Kaschuba W., "Kulturalismus: Kultur statt Gesellschaft? », Geschichte und Gesellschaft, vol. 21, $\mathrm{n}^{\circ} 1,1995$, pp. 80-95. 
